BIO Web of Conferences 2, 04003 (2014)

DOI: $10.1051 /$ bioconf/ 20140204003

(C) Owned by the authors, published by EDP Sciences, 2014

\title{
About the presence of arsenic in prebiotic species
}

\author{
Y. Ellinger ${ }^{1}$, M. Toulouze ${ }^{1}$, J. Pilmé $^{1}$ and F. Pauzat ${ }^{1}$ \\ ${ }^{1}$ Laboratoire de Chimie Théorique, UPMC (Univ. Paris VI), UMR-CNRS 7616, France
}

\begin{abstract}
The recent publication that some bacteria could use arsenic instead of phosphorus for building their DNA triggered a large controversy in the astro/exobiology community. Most comments claim that such a substitution is not possible. Here, we address the same question of the presence of As in DNA from a pure theoretical point of view, beyond any biological consideration. By means of "First principle" quantum calculations we found that there is no energetical or structural argument to reject the As to P substitution in the DNA helix. However, a topological analysis of the electron density shows that As-DNA is much more fragile and most probably will not survive because it lacks the covalent bonds that insure the stability of biological P-DNA.
\end{abstract}

\section{Introduction}

Since the publications of Wolfe-Simon reporting the replacement of phosphorus by arsenic in biomolecules of a bacterium $[1,2]$, numerous comments have claimed that such a substitution cannot be possible. Life indeed, as we know it today on Earth, is built on first row, C, H, O, N with second row $\mathrm{S}$ and $\mathrm{P}$ elements. In the cells of all the living organisms previously known on Earth, $\mathrm{P}$ is a vital element, forming a key component of biological molecules such as DNA. From a pure theoretical point of view, it is not a priori impossible that some other elements in the periodic table could serve the same functions; their discovery in prebiotic species, specifically those belonging to the third row or below in the periodic chart would open a new horizon in the search for new forms of life. The present report is a prospective study aimed at identifying if As can substitute for $\mathrm{P}$ (lighter element of the same column). As, indeed, is a chemical analog of $\mathrm{P}$ with a similar atomic radius, as well as near identical electronegativity.

\section{Principle and modeling}

Density Functional Theory (DFT) within the B3LYP formalism was used to assess the plausibility of the As to P substitution in a DNA helix. A comparative study of the Dimethylphosphate (DMP) and Dimethyl-arsenate (DMA) anions models was then carried out. All structures were fully optimized and verified to be true minima. All the energies reported ( $\mathrm{kcal} / \mathrm{mole}$ ) include Zero Point Energy corrections. The calculations were repeated in liquid water and in the ice for DMP and DMA, using the polarizable continuum model (PCM) with appropriate dielectric constants to simulate the environment [3] ie. 78.5 for liquid water and 600 for water ice at $(10-50 \mathrm{~K})$.

A topological analysis [4] of the Electron Localisation Function (ELF) has been carried out to analyze the evolution of the bonding properties in the DNA models. 


\section{Structure and energetics of DNA analogs}

The sugar-phosphate backbone of DNA possesses several unique attributes that make it especially suitable to carry nucleobases that form the basis of the genetic code. Among them is the ability to form a stable double helical architecture, which requires a highly specific conformational preference.

Dimethyl phosphate anion (DMP) has been known for a long time to be a representative model of the helical structure of DNA. Substitution of As to P gives three structures of Dimethyl arsenate anion (DMA) close to those of DMP. In addition to similar geometries these structures are found in the same stability ordering: $g g>t t>$ gt which means that the gauche/gauche isomer is the most stable one in both As and $\mathrm{P}$ series and should be the dominat structure according the minimum energy principle [5].
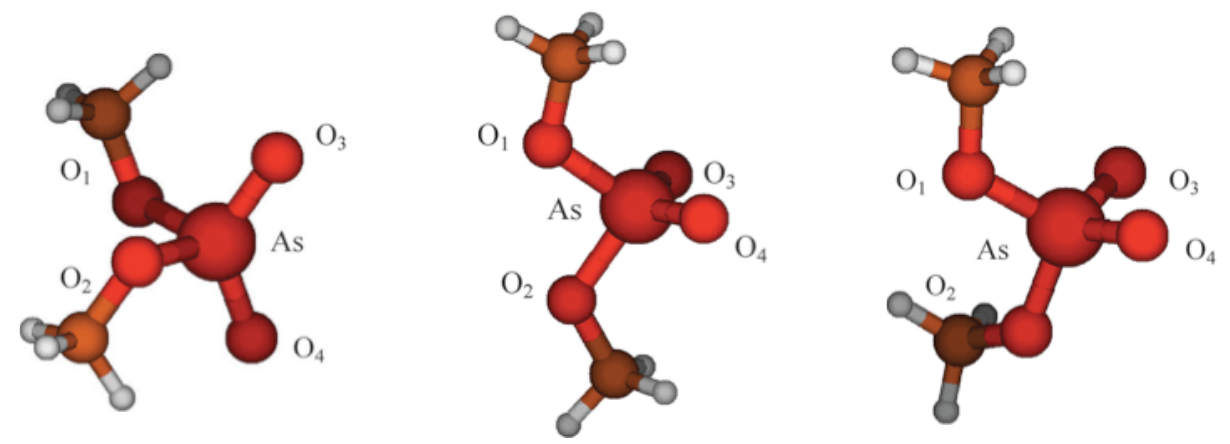

The gg, tt and gt conformations of the DMA anion model.

At this level of modeling, the simple DMP and DMA models both reproduce the characteristic helix form of DNA/RNA, suggesting that As can effectively replace $\mathrm{P}$ in the nuclear backbone. For a more realistic prebiotic picture, we considered DMA in a water-containing medium, i.e. in liquid water and in water ice. The net result is that hydration does not change the energetic order of the DMA structures. The difference in the stability between conformations is far greater than that for phosphorus, which may be limiting its flexibility.

However, the validity of the hypothesis of As replacing $\mathrm{P}$ in the prebiotic system cannot be assessed on structural similarities only. The topoplogical analysis of the ELF function allows a better insight at the electronic level going well beyond the strict structural/energetical comparison of the As and $\mathrm{P}$ series. The electronic population of the $\mathrm{As}-\mathrm{OCH}_{3}$ and $\mathrm{P}-\mathrm{OCH}_{3}$ bonds are 0.83 and $1.53 \mathrm{e}^{-}$ respectively whereas the populations of the $\mathrm{As}^{-} \mathrm{O}^{-}$and $\mathrm{P}_{-} \mathrm{O}^{-}$are 0.0 and 1.87 respectively, showing that the As-compound has a too weak backbone to maintain the appropriate [Ribose-O-P-O-Ribose-] structure to resist the conditions of life [6].

\section{References}

1. F. Wolfe-Simon, J. Switzer Blum, T.R. Kulp et al., Science, 332, 1163 (2011)

2. F. Wolfe-Simon, P.C.W. Davies et al., Int. J. Astrobiol, 8, 69 (2009)

3. J. Tomasi, M. Persico, Chem. Rev., 94, 2027 (1994)

4. B. Silvi, A. Savin, Nature, 371, 683 (1994)

5. M. Lattelais, F. Pauzat, Y. Ellinger, C. Ceccarelli, Astrophys. J., 696, L133 (2009)

6. M. Toulouze, J. Pilmé, F. Pauzat, Y. Ellinger, Phys. Chem. Chem. Phys., 14, 10516 (2012) 